\title{
VARIATION OF INSTANTANEOUS SPECTRAL CENTROID ACROSS BANDS OF SURFACE ELECTROMYOGRAPHIC SIGNALS
}

\author{
Divya Bharathi Krishnamani ${ }^{1}$, Karthick P.A. ${ }^{2}$, and Ramakrishnan Swaminathan ${ }^{1}$ \\ ${ }^{1}$ Non-Invasive Imaging and Diagnostics Laboratory, Biomedical Engineering Group, Department of \\ Applied Mechanics, Indian Institute of Technology Madras, Chennai, India \\ ${ }^{2}$ Physiological Measurements and Instrumentation Laboratory, Department of Instrumentation and \\ Control Engineering, National Institute of Technology Tiruchirappalli, Tiruchirappalli, India
}

Corresponding Author: Divya Bharathi Krishnamani

Email: divyak0593@gmail.com

https://doi.org/10.34107/BiomedSciInstrum.57.04356

\begin{abstract}
Surface electromyography (sEMG) is a technique which noninvasively acquires the electrical activity of muscles and is widely used for muscle fatigue assessment. This study attempts to characterize the dynamic muscle fatiguing contractions with frequency bands of sEMG signals and a geometric feature namely the instantaneous spectral centroid (ISC). The sEMG signals are acquired from biceps brachii muscle of fifty-eight healthy volunteers. The frequency components of the signals are divided into low frequency band $(10-45 \mathrm{~Hz})$, medium frequency band $(55-95 \mathrm{~Hz})$ and high frequency band $(95-400 \mathrm{~Hz})$. The signals associated with these bands are subjected to a Hilbert transform and analytical shape representation is obtained in the complex plane. The ISC feature is extracted from the resultant shape of the three frequency bands. The results show that this feature can differentiate the muscle nonfatigue and fatigue conditions $(p<0.05)$. It is found the values of ISC is lower in fatigue conditions irrespective of frequency bands. It is also observed that the coefficient of variation of ISC in the low frequency band is less and it demonstrates the ability of handling inter-subject variations. Therefore, the proposed geometric feature from the low frequency band of sEMG signals could be considered for detecting muscle fatigue in various neuromuscular conditions.
\end{abstract}

Keywords: Muscle fatigue, surface electromyography, frequency band analysis, geometric features, instantaneous spectral centroid

\section{INTRODUCTION}

Electromyography is a method that measures the electrical activity of skeletal muscles during varied contractions. The individual motor unit potentials can be measured accurately using needle electromyography which is invasive in nature. On the other hand, the surface electromyography (sEMG) technique noninvasively records the contractile activity from the skin surface. The sEMG signals produce the arithmetic summation of motor unit action potentials from the targeted muscle. It gains importance in the area of rehabilitation, sports medicine and fatigue analysis [1].

Muscle fatigue is a neuromuscular ailment that arises when there is temporary failure of muscles to produce maximal contractions. This leads to permanent damage of muscles if not diagnosed at an early stage [2]. Analysis of muscle fatigue using sEMG signals during isometric and dynamic contractions have been studied in recent years [3]. Separation of frequency bands in sEMG signals have also been attempted by several researchers for characterizing muscle fatigue [4]. These signals are highly complex in dynamic contractions due to continuous elbow-joint movement, velocity of movement and varied muscle length. The complexities such as nonstationarity, multiple frequency components and nonlinearity have been induced in sEMG signals while real-life dynamic activities are performed [5]. 